\section{Infection Control in a Rooming-In Environment}

\section{To the Editor:}

As Infection Control Coordinator of a small rural hospital, I am writing for any resource material or pertinent information on a problem I have encountered.

Our hospital has recently instituted a Rooming-In policy for obstetrical patients and their infants. Our policy states that one designated person is to be allowed in the room with the mother and infant. This person is to wear a gown and mask at all times while in the room. This is causing a problem and there has been poor adherence to this policy.

I would appreciate any information in regard to Infection Control in a Rooming-In Environment.

CAROLYN ADAMS, R.N. Infection Control Coordinator Memorial Hospital of Center Center, Texas

Ms. Adams' letter was referred to Dr. Timothy $R$. Townsend for a reply.

Rooming-in is one of several recent changes in perinatology. Slightly over 100 years ago, women avoided delivering their babies in hospitals because of the high risk of infection. As hospital deliveries became safer by the mid-1900s home deliveries became rare. Advances in monitoring techniques, in understanding fetal and infant physiology, and in the development of regionalization of perinatal services have led to a continued reduction in fetal wastage and infant mortality. These changes have focused on the physical wellbeing of both mother and child. In the mid-1940s at Yale University the first rooming-in facility was developed, primarily in response to perceived unmet emotional and psychological needs of both mother and child.' It was felt that if mother, father, and siblings could touch, cuddle, feed, and clothe the newborn, the emotional benefits to all concerned would be enhanced However, experience over the past century has taught that touching and cuddling newborns is precisely the mechanism by which some newborns acquire certain infections. This conflict of interest between the physical and psychological well-being of the newborn has led to confusion regarding the risk/benefit of rooming-in and very little data are available to help resolve the conflict. In the absence of data, most hospitals adopt a compromise position of allowing families to touch and cuddle their newborn infant but only after they have washed their hands and put on a gown.

The American Academy of Pediatrics (AAP) has recommended just such a position on rooming-in ("Standards and Recommendations for Hospital Care of Newborn Infants" published by the AAP to which inquiries regarding copies should be addressed; P.O. Box 1034, Evanston, IL 60204). In brief, the AAP recommends that the father or other person of the mother's choosing has, within limits of the needs of other mothers if multi-bed rooming-in facilities are used, unlimited visiting privileges. Unless rooming-in is intermittent, whereby the infant is removed from the room during visiting hours, other visiting is limited to one visitor in the room at a time. (I interpret this to mean father plus one other visitor at a time.) No one except the mother, the father, another person of the mother's choosing, and the hospital staff should handle the infant. All who handle the infant should wash their hands. It is recommended that long sleeve gowns be available but the AAP does not specifically indicate that the gowns should be worn by all persons at all times while in the room. I think it makes sense to wear a gown while handling an infant, but I see little benefit to wearing a gown just to sit in a room. Masks are not recommended for hospital personnel performing routine nursery activities so it makes little sense to recommend that rooming-in visitors wear them (the AAP does not address the "mask for rooming-in visitors" issue but makes a general statement regarding nursery attire). Finally, the AAP recommends that care for a rooming-in infant be of the same quality as that provided to an infant in the nursery and the same techniques and precautions to ensure the safety of infants be used regardless of their location within the hospital.

I think the AAP recommendations are reasonable and in the absence of better data concerning specific rooming-in infection control activities it seems wise to adopt the AAP recommendations. Overly stringent precautions do not seem warranted because only the healthiest of newborns should room-in and after only a few days the baby is home being kissed and cuddled by the extended family, friends, and occasionally perfect strangers. Somehow they survive it all.

\section{REFERENCE}

1. Jackson EB, Olmsted RW, Foord A, et al: A hospital rooming-in unit for four newborn infants and their mothers. Pediatrics 1948; 1:28-48.

TIMOTHY R. TOWNSEND, M.D. Hospital Epidemiologist The Johns Hopkins Hospital Baltimore, Maryland 